\title{
Transperitoneal Versus Extraperitoneal Laparoscopic Lymphadenectomy for Gynecological Malignancies: A Systematic Review and Meta-analysis
}

\author{
ANASTASIA PRODROMIDOU ${ }^{1}$, NIKOLAOS MACHAIRAS ${ }^{1}$, ELEFTHERIOS SPARTALIS ${ }^{1}$, \\ IOANNIS D. KOSTAKIS ${ }^{1}$, CHRISTOS IAVAZZO ${ }^{2}$, DIMITRIOS MORIS ${ }^{1}$, \\ DIAMANTIS I. TSILIMIGRAS ${ }^{1}$, ANTONIOS ATHANASIOU ${ }^{1}$ and NIKOLAOS NIKITEAS ${ }^{1}$ \\ ${ }^{1}$ Laboratory of Experimental Surgery and Surgical Research, Medical School, \\ National and Kapodistrian University of Athens, Athens, Greece; \\ ${ }^{2}$ Gynaecological Oncology Department, Metaxa Cancer Hospital, Piraeus, Greece
}

\begin{abstract}
Background/Aim: Para-aortic lymphadenectomy is established as a standard procedure for disease staging and management of patients with advanced cervical, ovarian and high-risk endometrial cancer. Our meta-analysis accumulated the current evidence on the impact of transperitoneal (TLL) and extraperitoneal (ELL) laparoscopic lymphadenectomy for the management of patients with gynecological malignancies. Materials and Methods: A systematic search of the literature was undertaken for articles published up to April 2018. Statistical meta-analysis was performed using the RevMan 5.3 software. Results: Seven studies were eligible for meta-analysis and comprised a total of 608 women. ELL was associated with significantly shorter operative times for lymphadenectomy, whereas total operative times were not different between the two groups. Significantly increased intraoperative complications were found in the TLL group. Conclusion: ELL is safe and efficient with outcomes comparable to TLL in terms of lymph node yield and improved operative outcomes with regard to lymphadenectomy times and intraoperative complications.
\end{abstract}

Para-aortic lymphadenectomy is indicated in patients with advanced cervical, ovarian and high-risk endometrial cancer. Lymph node dissection is essentially useful in order to tailor the optimal treatment of those patients according to their stage, provide adjuvant therapy in those with infiltrated lymph nodes and eliminate recurrence risk (1). Clinical

Correspondence to: Anastasia Prodromidou, MD, Laboratory of Experimental Surgery and Surgical Research, National and Kapodistrian University of Athens, Medical School, Ag. Thoma 15b, 11527, Athens, Greece. E-mail: a.prodromidou@ hotmail.com

Key Words: Extraperitoneal, transperitoneal, lymphadenectomy, minimally invasive, gynecological malignancies, meta-analysis. staging with imaging attains limited efficacy in accurately predicting lymph node involvement. On the other hand, surgical staging can provide accurate information concerning the exact extent of the disease and can result in survival benefit for the patient (1).

During the past decades, minimally-invasive techniques, albeit initially being considered technically complex, have become the standard of care; they have been proven safe and feasible with reduced morbidity, shorter length of hospital stay and prompt return to normal activity (2). Transperitoneal laparoscopic lymphadenectomy (TLL) has been widely adopted by proficient surgical teams for staging of gynecological malignancies (3). The extraperitoneal laparoscopic lymphadenectomy (ELL) approach has been proven to be an efficient alternative, also widely adopted, and has equally been associated with favorable outcomes. The objective of the present meta-analysis was to accumulate the current evidence with the intent to identify the impact of TLL and ELL for the management of patients with gynecological malignancies.

\section{Materials and Methods}

Study design. The present meta-analysis was designed according to the Preferred Reporting Items for Systematic Reviews and Meta-Analyses (PRISMA) guidelines (4) based on the Authors' predetermined eligibility criteria. Three Authors independently searched the literature (AP, NM, IDK). No language restrictions were applied. Prospective and retrospective clinical trials reporting outcomes for $>20$ women undergoing TLL versus ELL for treating or staging gynecological malignancies and reporting at least one postoperative outcome (operative time, blood loss, length of stay, postoperative complications, number of pelvic and para-aortic lymph nodes resected and cases of positive nodes) were considered eligible for inclusion. All discrepancies during the data collection, synthesis, and analysis were resolved by the consensus of all Authors. 
Literature search and data collection. A systematic search of the Medline, Scopus, Google Scholar, Cochrane CENTRAL Register of Controlled Trials and Clinicaltrials.gov databases for articles published up to April 2018 was performed, along with the references of all articles that were retrieved in full text. The performed search included the words "laparoscopic", "minimally invasive," "lymphadenectomy," "gynecological malignancies," "transperitoneal,", "extraperitoneal" and "retroperitoneal" (Figure 1).

The quality of all the included studies was assessed using the Methodological Index for Non-Randomized Studies (MINORS) (5). The MINORS scale was used due to the fact that all except one of the studies included in our meta-analysis were non-randomized.

Statistical analysis. Statistical meta-analysis was performed using the RevMan 5.3 software (Copenhagen: The Nordic Cochrane Centre, The Cochrane Collaboration, 2011). Confidence intervals (CI) were set at $95 \%$. Mean difference (MD) and odds ratios (OR) were used in the analysis. The results were calculated using the DerSimonianLaird random-effects model revealing significant heterogeneity in the methodological characteristics of the included studies (6). The cut-off for statistical significance was set at $p<0.05$. Mean values and standard deviations were calculated according to the equations proposed by Hozo et al. (7). Publication bias was not tested due to heterogeneity of the included studies, which is a confounder that may influence the methodological integrity of these tests.

\section{Results}

Excluded studies. Five studies were excluded from the present analysis. One reported outcomes for patients who underwent extraperitoneal and transperitoneal lymphadenectomy through laparotomy (8). Cartron et al. presented patients with pelvic and para-aortic lymphadenectomies but the reported outcomes were not considered eligible for inclusion (9). Another study was excluded since a single-port extraperitoneal approach was described (10), while Benito et al. did not present comparison outcomes between the two approaches (11). Finally, Dargent et al. included a limited number of patients (12).

Included studies. A total of seven studies, one randomized control trial and six retrospective trials, which included 608 patients who underwent para-aortic TLL versus ELL, were finally included in the present systematic review (13-19). Among them, 329 patients underwent TLL, while the remaining 279 patients comprised the ELL group. Six studies included cases in which laparoscopic procedures were performed, while in one study, procedures in both groups were either robotic-assisted or laparoscopic. Table I presents the main characteristics of the included studies and patients.

Quality assessment. The quality assessment using the MINORS scale showed that the included studies were methodologically adequate, with low heterogeneity regarding their quality, providing a mean score of $17.86(\mathrm{SD}=1.86)$ and a median score of 17 (range=17-22) (Table I).

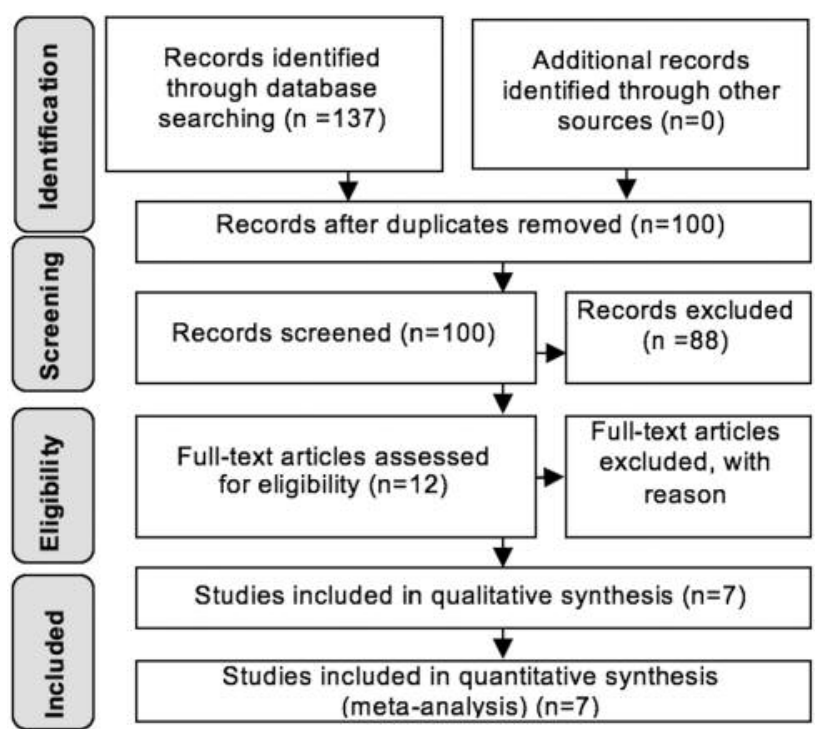

Figure 1. Search flow diagram.

Operative outcomes. Examination of intraoperative outcomes revealed no significant difference in total operative time between groups (407 patients: $\mathrm{MD}=-10.43 \mathrm{~min}, 95 \% \mathrm{CI}=-$ 20.55-41.42, $p=0.51)$. Interestingly, when lymphadenectomy time was separately analyzed, a significantly prolonged operative time was observed for the TLL group when compared with the ELL group (284 patients: $\mathrm{MD}=35.18 \mathrm{~min}$, $95 \% \mathrm{CI}=5.59$ to $64.76, p=0.02$ ) (Figure 2A). Furthermore, neither blood loss nor blood transfusion rates were found to differ between the two groups (218 patients: $\mathrm{MD}=64.90 \mathrm{ml}$, 95\% $\mathrm{CI}=-8.90-138.69, p=0.08$; and 403 patients: $\mathrm{OR}=1.39$, 95\% CI $=0.51-3.78, p=0.52$, respectively). Postoperative complications did not differ between the two groups (351 patients: $\mathrm{OR}=1.65,95 \% \mathrm{CI}=0.92-2.95, p=0.09)$. The TLL group presented a significantly higher rate of intraoperative complications (536 patients: $\mathrm{OR}=2.40,95 \% \mathrm{CI}=1.02-5.63$, $p=0.04$ ) (Figure 2B). No difference in terms of hospital stay between the two approaches was shown (433 patients: $\mathrm{MD}=0.35 \mathrm{~min}, 95 \% \mathrm{CI}=-0.77-1.48, p=0.54)$. Finally, mean number of resected lymph nodes was not different neither for pelvic nodes nor for para-aortic (300 patients: $\mathrm{OR}=-0.03,95 \%$ $\mathrm{CI}=-1.71-1.64, p=0.97$; and 479 patients: $\mathrm{OR}=-0.94,95 \%$ $\mathrm{CI}=-4.29-2.41, p=0.58$, respectively).

\section{Discussion}

Our study aimed to review and evaluate critical differences between TLL and ELL in patients with gynecological malignant lesions. The outcomes of the present meta-analysis demonstrated comparable total operative times, blood loss, blood transfusion rates, postoperative complication rates, 

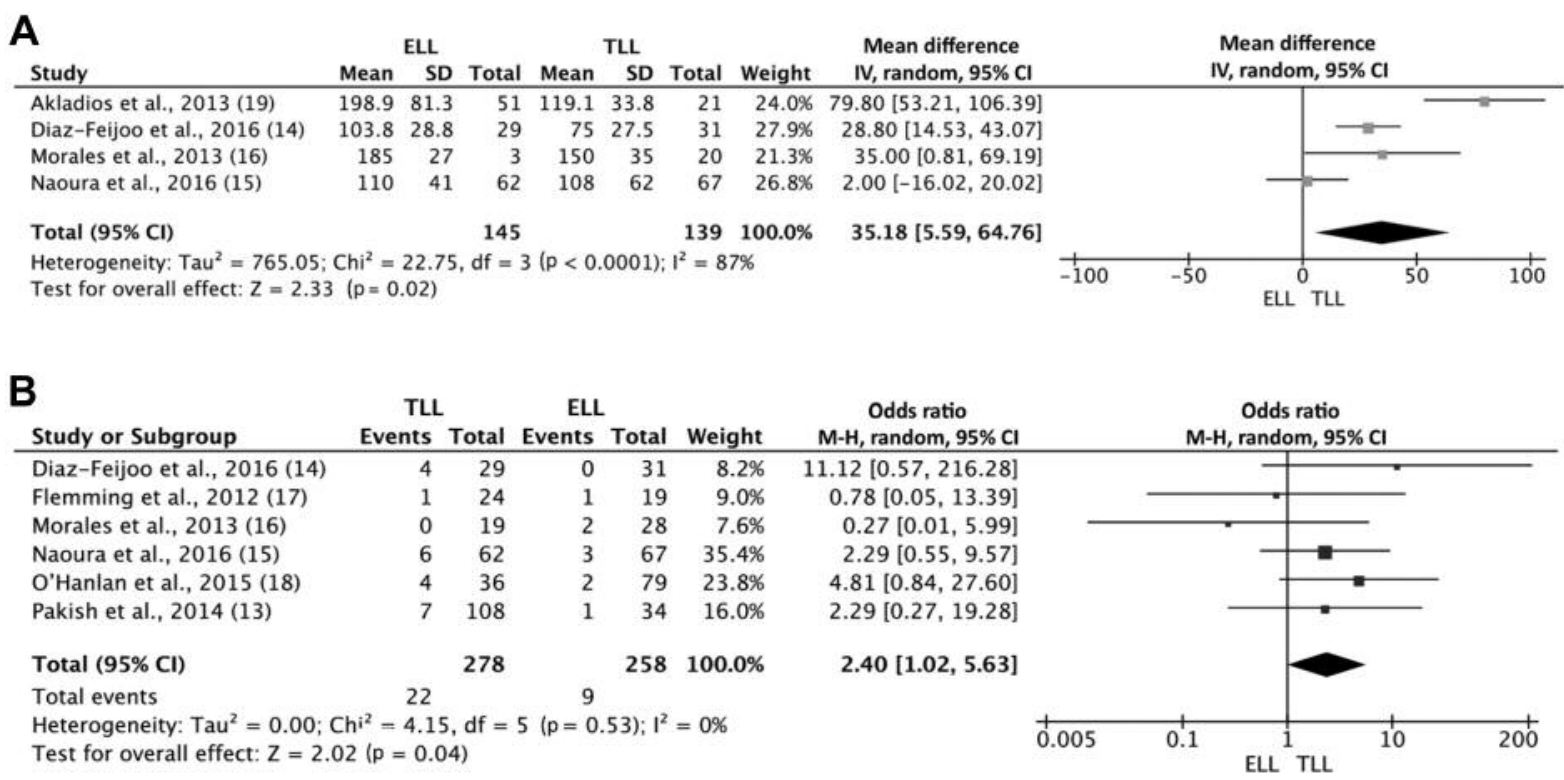

Figure 2. Forest plots depicting lymphadenectomy operative times (A) and intraoperative complications (B) during transperitoneal (TLL) and extraperitoneal (ELL) laparoscopic lymphadenectomy.

Table I. Characteristics of the included studies and patients.

\begin{tabular}{|c|c|c|c|c|c|c|}
\hline Author, year (ref) & Country & $\begin{array}{l}\text { Type of } \\
\text { study }\end{array}$ & MINORS & $\begin{array}{l}\text { No. of } \\
\text { patients }\end{array}$ & $\begin{array}{c}\text { Type of } \\
\text { malignancy (n) }\end{array}$ & $\begin{array}{c}\text { Type of } \\
\text { lymphadenectomy }\end{array}$ \\
\hline Diaz-Feijoo et al., 2016 (14) & Spain \& USA & Prospective RCT & 22 & 29 vs. 31 & $\begin{array}{c}\text { Endometrial: } 22 \text { vs. } 26 \\
\text { Ovarian: } 7 \text { vs. } 5\end{array}$ & $\begin{array}{l}\text { Para-aortic supra-and } \\
\text { inframesenteric }\end{array}$ \\
\hline Naoura et al., 2016 (15) & France & Retrospective & 17 & 62 vs. 67 & $\begin{array}{l}\text { Cervical: } 22 \text { vs. } 41 \\
\text { Endometrial: } 29 \text { vs. } 22 \\
\text { Ovarian: } 11 \text { vs. } 1 \\
\text { Vaginal: } 0 \text { vs. } 3\end{array}$ & Para-aortic \\
\hline O’Hanlan et al., 2015 (18) & USA & Retrospective & 18 & $36 v s .79$ & $\begin{array}{c}\text { Cervical: } 0 \text { vs. } 4 \\
\text { Endometrial: } 22 \text { vs. } 53 \\
\text { Tubal/ovarian: } 16 \text { vs. } 20\end{array}$ & $\begin{array}{l}\text { Para-aortic up to } \\
\text { infra-renal aorta }\end{array}$ \\
\hline Pakish et al., 2014 (13) & USA & Retrospective & 17 & 108 vs. 34 & Endometrial 108 vs. 34 & $\begin{array}{l}\text { Para-aortic up to } \\
\text { the renal vessels }\end{array}$ \\
\hline Akladios et al., 2013 (19) & France & Retrospective & 17 & 51 vs. 21 & $\begin{array}{l}\text { Cervical: } 22 \text { vs. } 16 \\
\text { Ovarian: } 20 \text { vs. } 3 \\
\text { Uterus: } 8 \text { vs. } 1\end{array}$ & $\begin{array}{l}\text { Trasnperitoneal: up to } \\
\text { the inferior mesenteric } \\
\text { artery Extraperitoneal: } \\
\text { up to the left renal vein }\end{array}$ \\
\hline Morales et al., 2013 (16) & Spain \& Germany & Retrospective & 17 & 19 vs. 28 & $\begin{array}{c}\text { Endometrial: } 9 \text { vs. } 6 \\
\text { Ovarian/fallopian: } 7 \text { vs. } 3 \\
\text { Cervical: } 3 \text { vs. } 19\end{array}$ & $\begin{array}{l}\text { Para-aortic up to the } \\
\text { level of renal vessels }\end{array}$ \\
\hline Fleming et al., 2012 (17) & USA & Retrospective & 17 & 24 vs. 19 & Endometrial & $\begin{array}{l}\text { Para-aortic Inferior } \\
\text { mesenteric artery }\end{array}$ \\
\hline
\end{tabular}

RCT: Randomized control trial; MINORS: Methodological Index for Non-Randomized Studies (5).

lengths of hospital stay and number of resected lymph nodes between the two groups. Nonetheless, the ELL group presented more favorable outcomes in terms of shorter lymphadenectomy times and fewer intraoperative complications.
Despite the fact that several studies have reviewed the outcomes of TLL or ELL for gynecological malignancies (3, 20,21 ), to our knowledge, the present study is the first to present outcomes on the comparison between them. The 
findings of the present meta-analysis indicate a potential beneficial effect of ELL with respect to operative time. Despite the fact that total operative time was not found to be different between the two groups, analysis of outcomes from studies which reported lymphadenectomy times separately revealed significantly protracted times in TLL. This outcome illustrates that when excluding the times required for performing the procedures additional to lymphadenectomy (e.g. hysterectomy, or omentectomy), patients who undergo the ELL approach could benefit from the shorter duration of surgery with regard to less exposure to anesthesia-related complications, with lower operative costs. Regardless, it should be noted that no difference in the total number of resected lymph nodes was observed between the two groups.

Despite the fact that lymphocele is reported as the most common postoperative complication related to lymphadenectomy (22), only two of the included studies separately presented postoperative incidence of lymphocele. Morales et al. reported one patient with lymphocele in the ELL group (16), whereas Naoura et al. found no difference in the proportion of patients diagnosed with lymphocele between the two groups (17\% vs. 7\%, $p=0.06)$ (15). Postoperative lymphedema rates were not reported separately by the included studies.

TLL is considered the optimal approach in patients with gynecological malignancies. However, the ELL approach is considered a beneficial alternative in the hands of experienced teams. The ELL technique provides a more secure access to the left aortic lymph nodes and specifically to challenging-to-reach supramesenteric lymph nodes. Additionally, the bowel does not interfere with the surgical field and the left ureter is always in sight and thus reduces the risk of injury to these structures (21). The ELL approach eliminates the risk of postoperative abdominal hernia and intra-abdominal adhesions, since the peritoneal cavity remains intact (23). Patients who have undergone previous multiple abdominal surgical procedures might theoretically benefit from the ELL approach in terms of reduced operative time due to avoidance of adhesiolysis, elimination of risk of intra-abdominal organ injury, and reduced blood loss and operative time. Furthermore, the extraperitoneal approach is specifically useful in obese patients due to the avoidance of the thick mesenteric adipose tissue of the small bowel and colon from the surgical field. On the other hand, ELL has been associated with longer learning curves; since the TLL technique reproduces the traditional open approach, surgeons are more familiar with the technique and it is most widely used. The fact that in the transperitoneal approach four trocars are traditionally utilized and two assistants are required, while in the extraperitoneal one three trocars and one assistant are needed is a matter of controversy. Akladios et al. demonstrated that despite the fact that ELL seems beneficial regarding the number of trocars and surgeons, due to the way that the trocars are placed in TLL, better management of potential complications was achieved (19).

Nevertheless, our study presents limitations. The retrospective nature of the majority of the included studies and their heterogeneity constitute a potential weakness; all the included studies except one were non-randomized, which limits evaluation of the exact role of patient characteristics such as body mass index as confounders. Therefore, our results may be subject to potential bias (selection bias, selective reporting and attrition bias) despite the fact that funnel plots of the examined parameters seem to be symmetrical. Furthermore, the limited number of the included studies, as well as the small sample sizes of the included patients in each group, constituted a further limitation of our study. The included studies did not report separate outcomes for each type of cancer, which represents a potential confounder in evaluating oncologicaI outcomes. Inadequate reporting of the exact level of lymph node dissection and exact definition of pelvic and para-aortic lymphadenectomy limits the exact interpretation of our findings.

\section{Conclusion}

ELL is a feasible and safe approach, which presents outcomes comparable to those of TLL in terms of lymph node yield and improved operative outcomes, with regard to lymphadenectomy times and intraoperative complications. Such results, however, must be interpreted with caution due to the limited number of studies in this field and their retrospective nature. Further larger-volume studies are needed in this field in order to define the optimal lymphadenectomy approach in patients with gynecological malignancies.

\section{Conflicts of Interest}

The Authors declare no conflicts of interest in regard to this study.

\section{References}

1 Marnitz S, Kohler C, Roth C, Fuller J, Hinkelbein W and Schneider A: Is there a benefit of pretreatment laparoscopic transperitoneal surgical staging in patients with advanced cervical cancer? Gynecol Oncol 99: 536-544, 2005.

2 Xiao $\mathrm{M}$ and Zhang Z: Total laparoscopic versus laparotomic radical hysterectomy and lymphadenectomy in cervical cancer: An observational study of 13-year experience. Medicine 94: e1264, 2005.

3 Kehoe SM and Abu-Rustum NR: Transperitoneal laparoscopic pelvic and paraaortic lymphadenectomy in gynecologic cancers. Curr Treat Options Oncol 7: 93-101, 2006.

4 Liberati A, Altman DG, Tetzlaff J, Mulrow C, Gotzsche PC, Ioannidis JP, Clarke M, Devereaux PJ, Kleijnen J and Moher D: The PRISMA statement for reporting systematic reviews and meta-analyses of studies that evaluate healthcare interventions: explanation and elaboration. BMJ 339: b2700, 2009. 
5 Slim K, Nini E, Forestier D, Kwiatkowski F, Panis Y and Chipponi J: Methodological index for non-randomized studies (minors): development and validation of a new instrument. ANZ J Surg 73: 712-716, 2003.

6 DerSimonian R and Kacker R: Random-effects model for metaanalysis of clinical trials: an update. Contemp Clin Trials 28 : 105-114, 2007.

7 Hozo SP, Djulbegovic B and Hozo I: Estimating the mean and variance from the median, range, and the size of a sample. BMC 5: $13,2005$.

8 Weiser EB, Bundy BN, Hoskins WJ, Heller PB, Whittington RR, DiSaia PJ, Curry SL, Schlaerth $\mathrm{J}$ and Thigpen JT: Extraperitoneal versus transperitoneal selective paraaortic lymphadenectomy in the pretreatment surgical staging of advanced cervical carcinoma (a Gynecologic Oncology Group study). Gynecol Oncol 33: 283-289, 1989.

9 Cartron G, Leblanc E, Ferron G, Martel P, Narducci F and Querleu D: Complications of laparoscopic lymphadenectomy in gynaecologic oncology. A series of 1102 procedures in 915 patients. Gynecol Obstet Fertil 33: 304-314, 2005.

10 Beytout C, Laas E, Naoura I, Bendifallah S, Canlorbe G, Ballester M and Daraï E: Single-port extra-and transperitoneal approach for paraaortic lymphadenectomy in gynecologic cancers: A propensity-adjusted analysis. Ann Surg Oncol 23: 952-958, 2016.

11 Benito V, Romeu S, Esparza M, Carballo S, Arencibia O, Medina $\mathrm{N}$ and Lubrano A: Safety and feasibility analysis of laparoscopic lymphadenectomy in pelvic gynecologic malignancies: A prospective study. Int J Gynecol Cancer 25: 1704-1710, 2015.

12 Dargent D, Ansquer Y and Mathevet P: Technical development and results of left extraperitoneal laparoscopic paraaortic lymphadenectomy for cervical cancer. Gynecol Oncol 77: 8792, 2000.

13 Pakish J, Soliman PT, Frumovitz M, Westin SN, Schmeler KM, Reis RD, Munsell MF and Ramirez PT: A comparison of extraperitoneal versus transperitoneal laparoscopic or robotic para-aortic lymphadenectomy for staging of endometrial carcinoma. Gynecol Oncol 132: 366-371, 2014.

14 Diaz-Feijoo B, Correa-Paris A, Perez-Benavente A, FrancoCamps S, Sanchez-Iglesias JL, Cabrera S, de la Torre J, Centeno C, Puig OP, Gil-Ibañez B, Colas E, Magrina J and Gil-Moreno A. prospective randomized trial comparing transperitoneal versus extraperitoneal laparoscopic aortic lymphadenectomy for surgical staging of endometrial and ovarian cancer: The STELLA trial. Ann Surg Oncol 23: 2966-2974, 2016.
15 Naoura I, Laas E, Beytout C, Bendifallah S, Ballester M and Darai E: A propensity score evaluation of single-port or multiport extraperitoneal para-aortic lymphadenectomy and the transperitoneal approach for gynecological cancers. Bull Cancer 103: 320-329, 2016.

16 Morales S, Zapardiel I, Grabowski JP, Hernandez A, Diestro MD, Gonzalez-Benitez C and de Santiago J: Surgical outcome of extraperitoneal paraaortic lymph node dissections compared with transperitoneal approach in gynecologic cancer patients. J Minim Invasive Gynecol 20: 611-615, 2013.

17 Fleming ND, Axtell AE and Lentz SE: Operative and anesthetic outcomes in endometrial cancer staging via three minimally invasive methods. J Robot Surg 6: 337-344, 2012.

18 O'Hanlan KA, Sten MS, O'Holleran MS, Ford NN, Struck DM and McCutcheon SP: Infrarenal lymphadenectomy for gynecological malignancies: two laparoscopic approaches. Gynecol Oncol 139: 330-337, 2015.

19 Akladios C, Ronzino V, Schrot-Sanyan S, Afors K, Fernandes R, Baldauf JJ and Wattiez A: Comparison between transperitoneal and extraperitoneal laparoscopic paraaortic lymphadenectomy in gynecologic malignancies. J of Minim Invasive Gynecol 22: 268-274, 2015.

20 Lecuru F and Taurelle R: Transperitoneal laparoscopic pelvic lymphadenectomy for gynecologic malignancies (I). Technique and results. Surg Endosc 12: 1-6, 1998.

21 Kusunoki S, Huang K-G, Magno A and Lee C-L: Laparoscopic technique of para-aortic lymph node dissection: A comparison of the different approaches to trans-versus extraperitoneal paraaortic lymphadenectomy. Gynecol Minim Invasive Ther 6: 5157, 2017.

22 Occelli B, Narducci F, Lanvin D, Coste E, Legoupils E, Castelain $\mathrm{B}$ and Querleu D: Comparison of transperitoneal versus extraperitoneal laparoscopic para-aortic lymphadenectomy: randomized experimental study. Ann Chir 125: 9-17, 2000.

23 Vasilev SA and McGonigle KF: Extraperitoneal laparoscopic para-aortic lymph node dissection. Gynecol Oncol 61: 315-320, 1996.
Received June 18, 2018

Revised June 27, 2018

Accepted July 2, 2018 